\title{
EFEITO DA TEMPERATURA DE RECOZIMENTO NA PRECIPITAÇÃO DA FASE SIGMA NO AÇO INOXIDÁVEL DUPLEX UNS 31803*
}

\section{Resumo}

Graziele Gianini Braga Maria ${ }^{1}$ Carolina Arriel Pedroso Dias ${ }^{2}$

Davi Silva Alves²

Aline Oliveira Vasconcelos Ferreira² lane Dutra Moutinho²

Aline Silva Magalhães ${ }^{3}$

Dagoberto Brandão Santos ${ }^{4}$

O aço inoxidável duplex combina as propriedades mecânicas da ferrita à resistência à corrosão da austenita, sendo muito utilizado em aplicações industriais que requerem um bom desempenho quanto a essas propriedades. Quando esse aço é submetido a ciclos térmicos, fases secundárias podem se precipitar, o que causa a deterioração de suas propriedades. Dentre as fases que podem surgir, a fase sigma é a de maior relevância. O presente trabalho tem como objetivo avaliar e quantificar a precipitação da fase sigma quando o aço duplex UNS 31803 é submetido ao recozimento em diferentes temperaturas. Para essa análise foram utilizados a microscopia óptica e eletrônica, difração por elétrons retroespalhados, difração de raios - $X$, e espectometria de raios - X por dispersão em energia. Observou - se a presença da fase sigma em três das quatro condições adotadas no trabalho. A presença mais acentuada da fase foi observada na condição em que a velocidade de precipitação é máxima.

Palavras-chave: Aço inoxidável duplex; Fase sigma; Fases intermetálicas.

\section{EFFECT OF ANNEALING TEMPERATURE ON THE PRECIPITATION OF SIGMA PHASE IN DUPLEX STAINLESS STEEL UNS 31803}

\section{Abstract}

The duplex stainless steel combines the mechanical properties of ferrite and the corrosion resistance of austenite, widely used in industrial applications that require a good performance in terms of these properties. When this steel is subjected to thermal cycles, secondary phases can precipitate, which causes the deterioration of its properties. The sigma phase is the most relevant between the precipitates which may be formed. The aim of this work is to evaluate and quantify the precipitation of sigma phase when the Duplex UNS 31803 steel is subjected to annealing at different temperatures. For this analysis were used optical and electron microscopy, electron backscattered diffraction, diffraction $X$ - ray spectrometry and $X$ - ray dispersive energy. The sigma phase was observed in three of the four conditions adopted in work. The presence of phase was observed so more pronounced in the condition where the precipitation velocity is maximal.

Keywords: Duplex stainless steel; Phase sigma; Phases intermetallic.

1 Eng. Materiais, ME, Doutoranda no Programa de Pós-Graduação em Engenharia Metalúrgica, Materiais e de Minas, UFMG, Belo Horizonte, MG, Brasil.

2 Graduando(a) Eng. Metalúrgica, Iniciação científica no Departamento de Engenharia Metalúrgica, UFMG, Belo Horizonte, MG, Brasil.

3 Eng. Materiais, ME, Doutoranda no Programa de Pós-Graduação em Engenharia Metalúrgica, Materiais e de Minas, UFMG, Belo Horizonte, MG, Brasil.

4 Eng. Metalurgista, Dr., Professor titular, Departamento de engenharia metalúrgica e de materiais, UFMG, Belo Horizonte, MG, Brasil. 


\section{INTRODUÇÃO}

Aços inoxidáveis duplex (AID) são ligas de microestrutura austeno $(\gamma)$ - ferritica $(\delta)$ desenvolvidas a partir do sistema $\mathrm{Fe}$ - $\mathrm{Cr}$ - Ni. Em geral, a proporção entre as fases é de $50 \%$, podendo variar de $30 \%$ a $70 \%$ de ferrita $[1,2,3,4]$. Para que essas proporções sejam atingidas, os aços duplex são submetidos à laminação a quente ou forjamento, laminação a frio, seguida de um recozimento para recristalização[5,6].

Quando comparados aos aços ferríticos e austeníticos, os AID's exibem melhores propriedades mecânicas e corrosivas, sendo utilizados nas indústrias petroquímica, químicas, óleo e gás, papel e celulose, naval e nuclear, as quais requerem boa combinação dessas propriedades [7-10].

Os aços duplex apresentam forte tendência à formação de fases secundárias quando expostos a altas temperaturas por tempos prolongados. Essa tendência é dependente dos elementos de liga presentes e pode variar entre os aços da classe [11,12]. A precipitação dessas fases ocorre entre $300^{\circ} \mathrm{C}$ e $1000^{\circ} \mathrm{C}$, conforme observado na Figura $1[11,13,14]$.

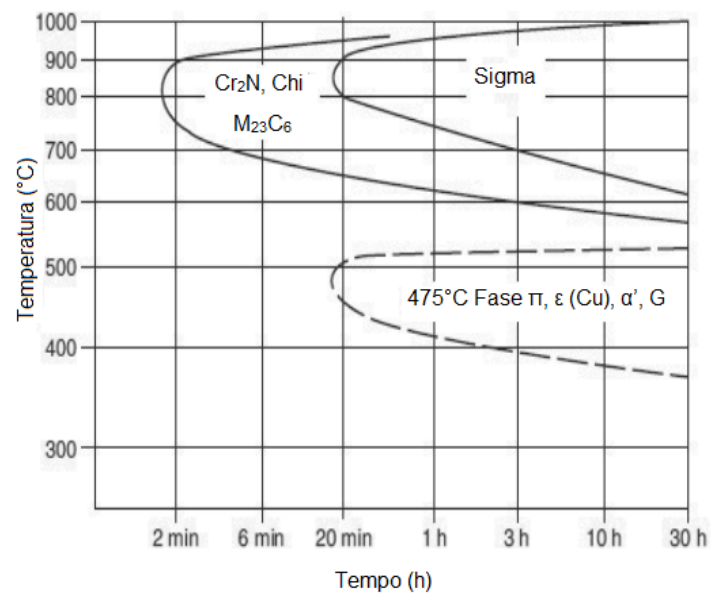

Figura 1. Diagrama TTT exibindo a precipitação das fases secundárias [15]

Entre as fases apresentadas no diagrama, a fase sigma $(\sigma)$ é a mais importante, em razão dos severos efeitos que sua presença pode causar nas propriedades dos aços [16]. A nucleação da fase $\sigma$ ocorre preferencialmente na interface $\delta / \delta$ e $\delta / \gamma$ entre $650^{\circ} \mathrm{C}$ e $950^{\circ} \mathrm{C}$ [15-18]. A concentração de Mo e $\mathrm{Cr}$ na fase $\sigma$ é maior em relação a ferrita, o que leva a um empobrecimento desses elementos na matriz, reduzindo a resistência a corrosão e tenacidade do material [19].

O objetivo desse artigo é avaliar quantitativamente a formação da fase sigma em um aço duplex submetido a laminação a frio e a recozimento subsequente em diferentes temperaturas.

\section{MATERIAIS E MÉTODOS}

Neste trabalho, utilizou-se o aço inox duplex UNS 31803 na forma de chapas de 4,0 $\mathrm{mm}$ de espessura. A composição química informada pelo fabricante pode ser visualizada na Tabela 1. 
Tabela 1. Composição química do aço UNS 31803

\begin{tabular}{cccccccccc}
\hline Elemento & $\mathrm{C}$ & $\mathrm{Mn}$ & $\mathrm{Si}$ & $\mathrm{Cr}$ & $\mathrm{Ni}$ & $\mathrm{P}$ & $\mathrm{S}$ & $\mathrm{Mo}$ & $\mathrm{Cu}$ \\
\hline $\begin{array}{c}\text { \% em } \\
\text { peso }\end{array}$ & 0,013 & 1,81 & 0,38 & 22,45 & 5,31 & 0,02 & 0,0005 & 2,63 & 0,24 \\
\hline
\end{tabular}

A laminação a frio foi realizada em um laminador de laboratório da marca Fröhling, a uma velocidade de $6,5 \mathrm{~m} / \mathrm{min}$. A redução na espessura foi de $60 \%$, alcançada em 7 passes. Executou-se o recozimento em um forno da marca Linn Elektro Therm, conforme condições apresentadas na Tabela 2. Após uma análise preliminar dos resultados, optou-se por realizar o tratamento a $850^{\circ} \mathrm{C} / 24 \mathrm{~h}$, com o objetivo de potencializar a precipitação da fase sigma.

Tabela 2. Condições de recozimento consideradas no trabalho

\begin{tabular}{cc}
\hline Temperaturas & Tempo de encharque $(\mathrm{h})$ \\
\hline $850^{\circ} \mathrm{C}, 900^{\circ} \mathrm{C}, 950^{\circ} \mathrm{C}$ & 0,5 \\
$850^{\circ} \mathrm{C}$ & 24 \\
\hline
\end{tabular}

A caracterização microestrutural foi conduzida por meio da microscopia óptica (MO) e de varredura por feixe de elétrons (MEV), e da técnica de difração por elétrons retroespalhados (EBSD). As amostras utilizadas na MO e MEV foram preparadas segundo o procedimento convencional, usando o reativo de Kallings para o ataque químico. As amostras para o EBSD foram preparadas seguindo o mesmo procedimento, com exceção da etapa de ataque químico, que foi substituída por uma etapa de polimento mecânico com sílica coloidal 0,05 $\mu \mathrm{m}$.

Uma análise química foi executada por meio do espectrômetro de raios - X por dispersão de energia (EDS).

A difração de raios - $X(D R X)$ foi realizada tendo como objetivo a identificação das fases presentes no aço. O ângulo de varredura empregado foi de $30^{\circ}$ a $120^{\circ} \mathrm{com}$ passo de $0,02 \mathrm{~s}^{-1}$. A preparação das amostras deu - se até a etapa de polimento. Obteve-se a quantificação das fases por meio do software OriginPro8, empregando o método de integração das áreas abaixo dos picos.

\section{RESULTADOS E DISCUSSÃO}

Na Figura 2, é exibida uma micrografia do aço UNS 31803, nas quatro condições de recozimento adotadas. As Figuras $2 \mathrm{a}-\mathrm{d}$ são relacionadas a $\mathrm{MO}$ e as Figuras $2 \mathrm{e}-\mathrm{h}$ à MEV. Pode-se observar uma microestrutura característica de um aço duplex: uma matriz ferrítica com ilhas de austenita [13]. Nas Figuras $2 e, f, h$ é notada a presença da fase sigma, destacada pelo círculo vermelho, o que não se pode observar na Figura $2 \mathrm{~g}$, relativa ao recozimento em $950^{\circ} \mathrm{C}$. Nas imagens feitas por meio da microscopia óptica, não é possível identificar a fase sigma de forma mais pronunciada. Isso ocorre em razão desta fase estar finamente dispersa na ferrita. 

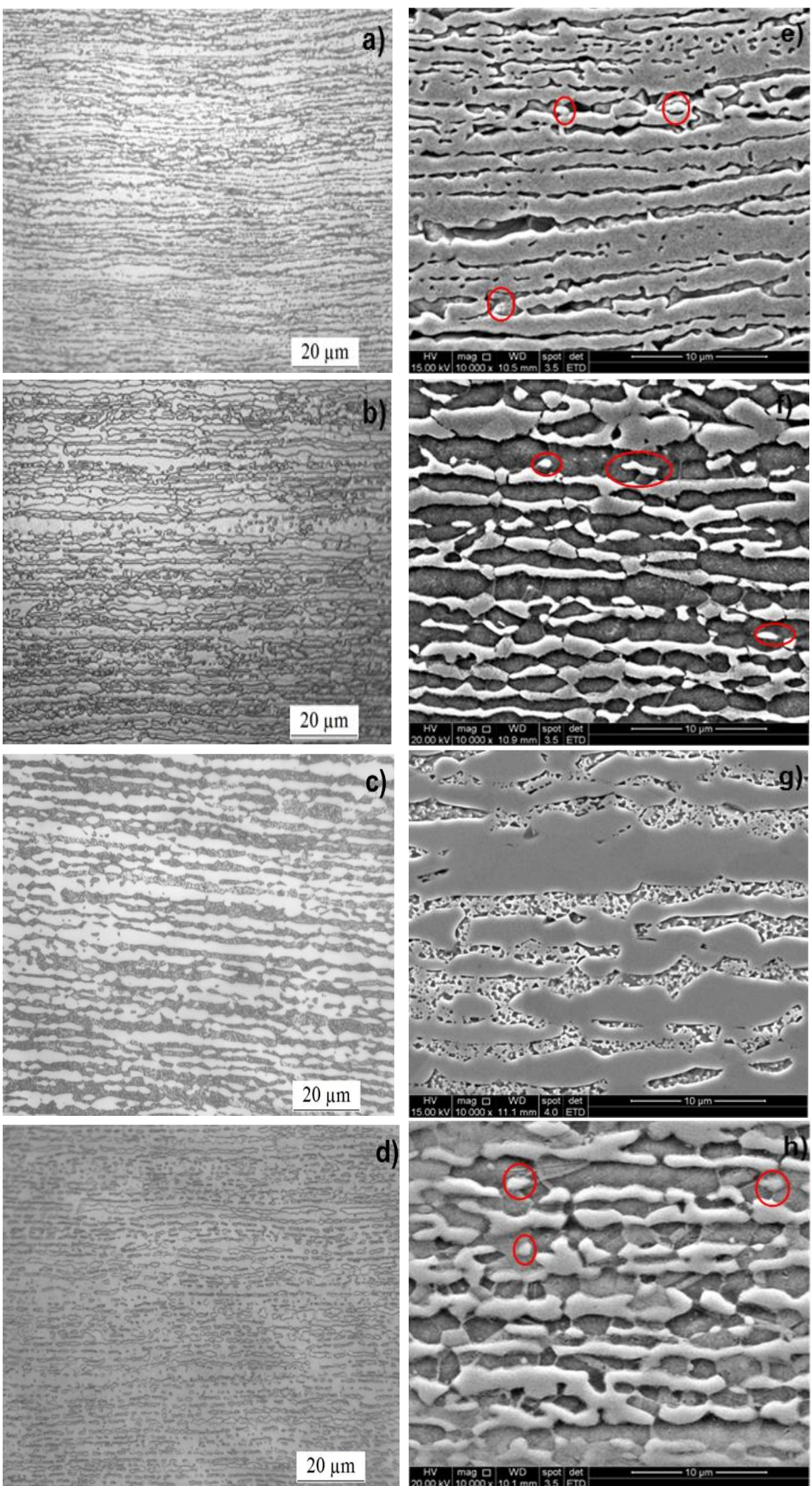

Figura 2. Fotomicrografia do aço 31803 recozido $850^{\circ} \mathrm{C} / 0,5 \mathrm{~h}$ (a) $\mathrm{MO}$ e (e) $\mathrm{MEV}, 900^{\circ} \mathrm{C} / 0,5 \mathrm{~h}$ (b) $\mathrm{MO}$ e (f) MEV, $950^{\circ} \mathrm{C} / 0,5 \mathrm{~h}$ (c) $\mathrm{MO}$ e (g) MEV, $850^{\circ} \mathrm{C} / 24 \mathrm{~h}$ (d) $\mathrm{MO}$ e (h) MEV. 
$\mathrm{Na}$ Tabela 3, a análise química geral e de cada fase em cada uma das condições adotadas no trabalho, pode ser verificada. A composição química fornecida pelo EDS corrobora a análise feita nas micrografias, uma vez que a composição química de $\sigma$ obtida pelo ensaio, $\mathrm{Fe} 27,3 \mathrm{Cr} 4,6 \mathrm{Ni}, 7 \mathrm{Mo}\left(850^{\circ} \mathrm{C} / 0,5 \mathrm{~h}\right), \mathrm{Fe} 31,5 \mathrm{Cr} 3,1 \mathrm{Ni}, 2 \mathrm{Mo}$ $\left(850^{\circ} \mathrm{C} / 24 \mathrm{~h}\right), \mathrm{Fe} 29,3 \mathrm{Cr} 4,1 \mathrm{Ni}, 6 \mathrm{Mo}\left(900^{\circ} \mathrm{C} / 0,5 \mathrm{~h}\right)$, está bem próxima à composição química de $\sigma$ encontrada na literatura, Fe30Cr4Ni4-7mo[4,9,19]. As microestruturas apontadas como fase sigma apresentam o teor de $\mathrm{Cr}$ e Mo maior do que o teor destes elementos na ferrita, o que também evidência a correta identificação da fase nas micrografias.

Tabela 3. Composição química da fase sigma fornecida pelo EDS

\begin{tabular}{|c|c|c|c|c|c|c|c|}
\hline & & $\mathrm{Si}$ & Mo & $\mathrm{Cr}$ & $\mathrm{Mn}$ & $\mathrm{Fe}$ & $\mathrm{Ni}$ \\
\hline \multirow{4}{*}{$\begin{array}{c}850^{\circ} \mathrm{C}-30 \\
\min \end{array}$} & Geral & 0,44 & 2,68 & 23,04 & 2,54 & 66,09 & 5,22 \\
\hline & Austenita & 0,65 & 2,11 & 21,61 & 2,32 & 65,27 & 8,05 \\
\hline & Ferrita & 0,55 & 2,03 & 22,98 & 2,03 & 67,17 & 5,24 \\
\hline & Sigma & 0,91 & 8,67 & 27,28 & 2,01 & 56,54 & 4,58 \\
\hline \multirow{4}{*}{$\begin{array}{c}900^{\circ} \mathrm{C}-30 \\
\min \end{array}$} & Geral & 0,59 & 3,00 & 24,14 & 1,59 & 64,83 & 5,86 \\
\hline & Austenita & 0,51 & 2,50 & 22,20 & 2,78 & 64,67 & 7,35 \\
\hline & Ferrita & 0,60 & 2,70 & 23,92 & 2,66 & 65,04 & 5,09 \\
\hline & Sigma & 0,88 & 6,61 & 29,32 & 1,97 & 57,11 & 4,11 \\
\hline \multirow{3}{*}{$\begin{array}{c}950^{\circ} \mathrm{C}-30 \\
\min \end{array}$} & Geral & 0,48 & 2,73 & 23,5 & 2,76 & 64,15 & 6,39 \\
\hline & Austenita & 0,40 & 2,49 & 21,52 & 1,80 & 65,22 & 8,56 \\
\hline & Ferrita & 0,76 & 4,03 & 26,21 & 2,11 & 62,11 & 4,79 \\
\hline \multirow{4}{*}{$850^{\circ} \mathrm{C}-24 \mathrm{~h}$} & Geral & 0,56 & 2,78 & 23,44 & 1,93 & 66,05 & 5,24 \\
\hline & Austenita & 0,64 & 2,58 & 22,06 & 2,10 & 66,61 & 6,01 \\
\hline & Ferrita & 0,59 & 1,47 & 20,46 & 2,27 & 67,88 & 7,33 \\
\hline & Sigma & 0,71 & 6,16 & 31,46 & 1,73 & 56,83 & 3,11 \\
\hline
\end{tabular}

Pode-se observar na Figura 3a-c os difratogramas de todas as condições analisadas no trabalho. Os picos característicos de cada fase estão indicados por seus respectivos símbolos, conforme padrão e a literatura [12, 20]. É possível notar a presença da fase sigma nas Figuras $3 a$ e $3 b$, 3d, as quais estão associadas aos recozimentos feitos a $850^{\circ} \mathrm{C}$ e $900^{\circ} \mathrm{C}$. O mesmo não pode ser observado na Figura 3c, na qual a amostra foi tratada a $950^{\circ} \mathrm{C}$. Outras fases secundárias, $\mathrm{Cr}_{2} \mathrm{~N}, \mathrm{Fe}$, $\mathrm{FeN}$,0056, carbonetos, também podem ser vistas nas Figuras 3a,b,d.

A capacidade de precipitação de $\sigma$ está relacionado à composição química da ferrita. Uma elevada presença de elementos formadores da fase sigma, $\mathrm{Cr}, \mathrm{Mo}, \mathrm{Si}$, assim como a escassez de elementos menos solúveis em $\sigma$, favorecem a formação da fase intermetálica analisada. Além da composição química, a reação eutetóide exibida na Equação 1 favorece o aparecimento de sigma. Para que a decomposição ocorra, é necessária a presença de elementos formadores da austenita, Ni, N, C. Quando comparada à austenita original, a austenita formada, $\gamma^{*}$, é escassa dos elementos $\mathrm{Cr}$ e Mo [21,22].

$$
\delta \rightarrow \gamma^{*}+\sigma
$$



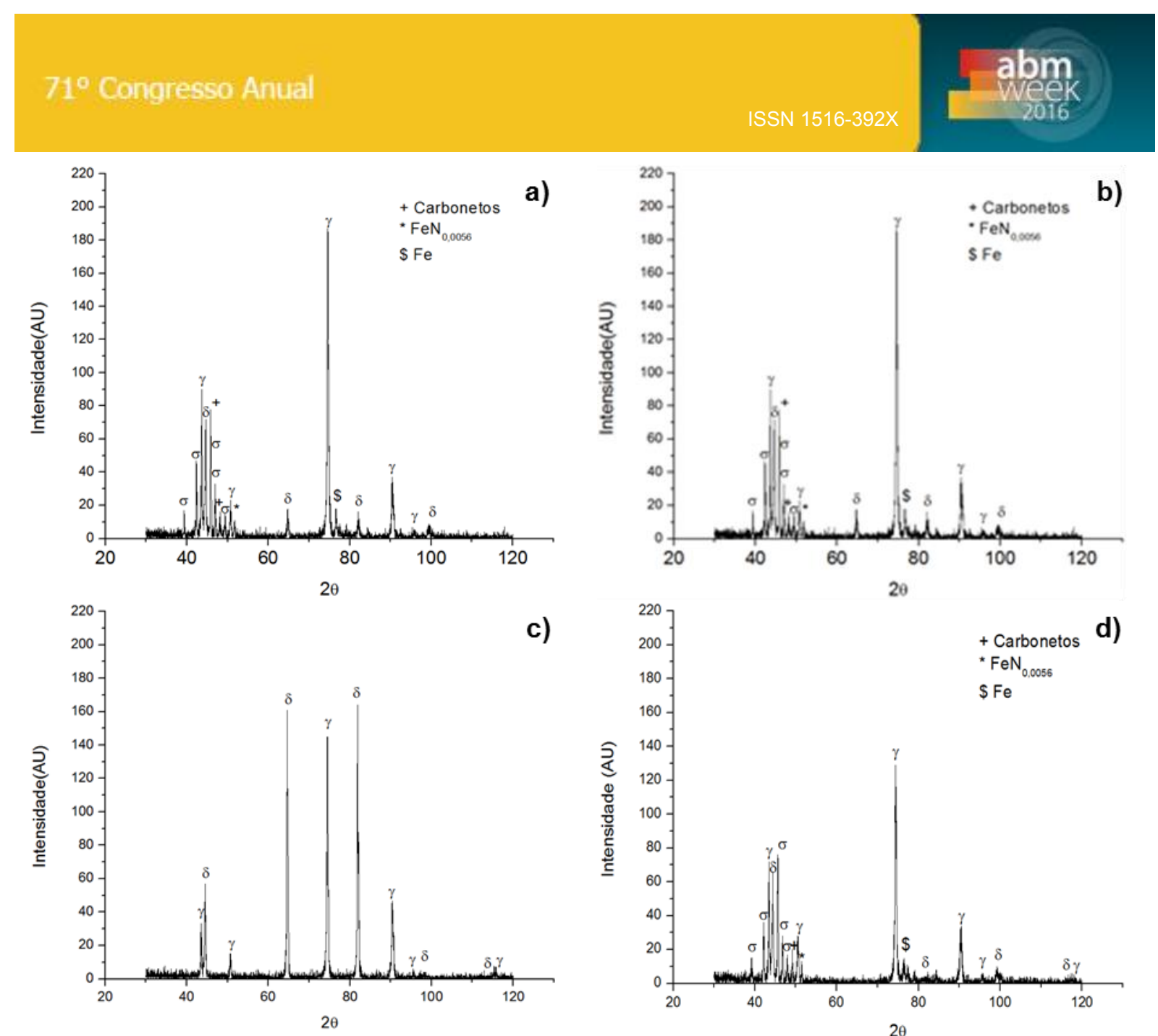

Figura 3. Difratogramas do aço UNS 31803 recozido a (a) $850^{\circ} \mathrm{C} / 0,5 \mathrm{~h}$, (b) $900^{\circ} \mathrm{C} / 0,5 \mathrm{~h}$, (c) $950^{\circ} \mathrm{C} / 0,5 \mathrm{~h}$, (d) $850^{\circ} \mathrm{C} / 24 \mathrm{~h}$

A Tabela 4 exibe a quantificação das fases identificadas em cada um dos difratogramas.

Tabela 4. Quantificação das fases (\%)

\begin{tabular}{c|cccc}
\hline & $850^{\circ} \mathrm{C} / 0,5 \mathrm{~h}$ & $900^{\circ} \mathrm{C} / 0,5 \mathrm{~h}$ & $950^{\circ} \mathrm{C} / 0,5 \mathrm{~h}$ & $850^{\circ} \mathrm{C} / 24 \mathrm{~h}$ \\
\hline $\mathrm{Y}$ & 61,9 & 61,8 & 43,5 & 59,4 \\
$\delta$ & 16,6 & 25,0 & 56,5 & 13,5 \\
Sigma & 9,3 & 4,8 & & 22,0 \\
Carboneto & 9,2 & 5,5 & & 1,6 \\
$\mathrm{Cr}_{2} \mathrm{~N}$ & & 2,9 & & \\
$\mathrm{Fe}$ & 2,1 & & & 2,1 \\
$\mathrm{X}$ & & & & 0,7 \\
FeN0,0056 & 1,0 & & & 0,7 \\
\hline
\end{tabular}

O recozimento feito a $850^{\circ} \mathrm{C}$ propiciou a formação de uma maior quantidade de fase $\sigma$ em relação ao tratamento feito a $900^{\circ} \mathrm{C}$, conforme apresentado na Tabela 4. Esse comportamento pode ser explicado em razão da velocidade de precipitação ser máxima em torno de $850^{\circ} \mathrm{C}$ [23]. Considerando o mesmo tempo de encharque, $0,5 \mathrm{~h}$, uma velocidade mais elevada favorece a formação de uma maior quantidade do precipitado. 
A condição de $950^{\circ} \mathrm{C}$, conforme observado na Tabela 4, não exibiu a presença de fases secundárias. A cinética de precipitação a $950^{\circ} \mathrm{C}$ é mais lenta quando comparado à velocidade de precipitação a $850^{\circ} \mathrm{C}$, sendo necessário um maior tempo para que as fases secundárias se precipitem.

No recozimento realizado $850^{\circ} \mathrm{C} / 24 \mathrm{~h}$ boa parte da ferrita foi convertida em fases secundárias, sendo sigma a fase predominante. Nessa condição foi identificado a presença de uma outra fase intermetálica, a fase Chi (X). A fase apresenta maior velocidade de precipitação, sendo nucleada nos primeiros minutos do recozimento. Tempos de encharque prolongados levam a transformação de chi em sigma [24,25]. Na Figura 4, a identificação e quantização das fases obtidas por meio da técnica EBSD são apresentadas. A amostra recozida à $850^{\circ} \mathrm{C} / 0,5 \mathrm{~h}$ apresentou uma maior quantidade de fase sigma quando comparada à condição de $900^{\circ} \mathrm{C} / 0,5 \mathrm{~h}$, conforme identificado na difração de raios - X. No entanto, a fração de sigma obtida pela DRX foi menor do que a fração indicada pelo EBSD. Essa diferença pode ser atribuída ao processamento de imagem feito pelo software OIM. Funções do software possibilitam que regiões de baixo índice de indexação sejam identificadas como pertencentes a uma determinada fase, o que altera a quantidade da mesma.
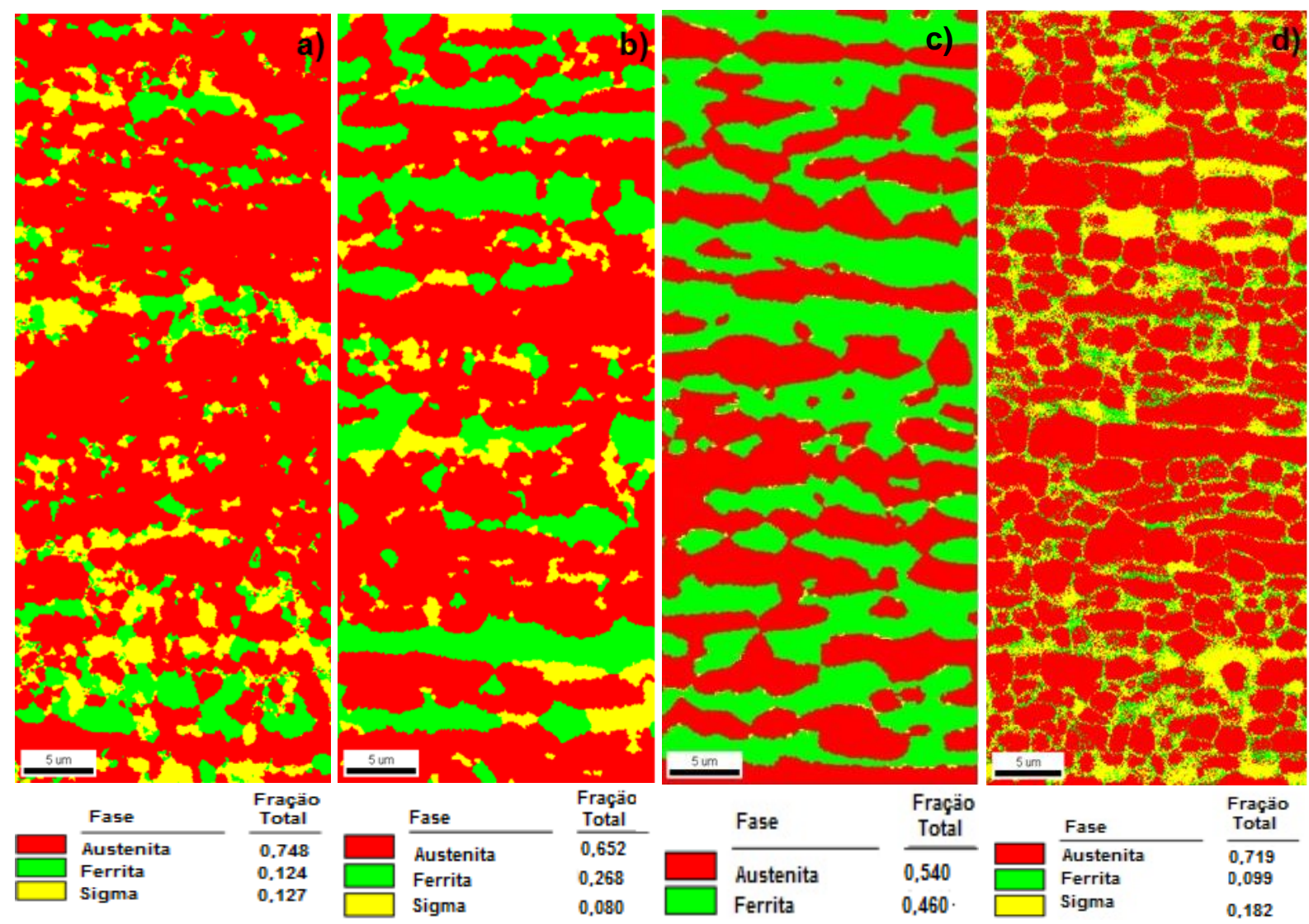

Figura 4. Mapa de fase obtido por meio do EBSD para a amostra recozida a (a) $850^{\circ} \mathrm{C} / 0,5 \mathrm{~h}$, (b) $900^{\circ} \mathrm{C} / 0,5 \mathrm{~h},(\mathrm{c}) 950 / 0,5 \mathrm{~h}$, (d) $850^{\circ} \mathrm{C} / 24 \mathrm{~h}$

Em geral a quantificação das fases obtida por meio das duas técnicas foi próxima, apresentando variação em torno de $4 \%$. As outras fases secundárias identificadas por meio do DRX não foram observadas no EBSD. Limitações do equipamento impossibilitaram a detecção dessas fases, que em geral se encontram finamente dispersas na matriz ferrítica. 


\section{CONCLUSÕES}

A cinética de precipitação de sigma é máxima à $850^{\circ}$, logo a condição de recozimento $850^{\circ} \mathrm{C} / 0,5 \mathrm{~h}$ apresentou maior quantidade de sigma quando comparada a condição $900^{\circ} \mathrm{C} / 0,5 \mathrm{~h}$.

A condição de $950^{\circ} \mathrm{C} / 0,5 \mathrm{~h}$ não possibilitou a precipitação da fase sigma, sendo necessário um maior tempo para que as fases secundárias se nucleiem.

Quanto maior o tempo a $850^{\circ} \mathrm{C}$ maior será a precipitação de sigma na matriz ferrítica, conforme observado no recozimento realizado a $850^{\circ} \mathrm{C} / 24 \mathrm{~h}$.

Outras fases secundárias foram observadas por meio da difração de raios - x, porém em virtude da limitação das outras técnicas de caracterização, não foram encontradas outras evidências de sua presença.

\section{Agradecimentos}

Os autores agradecem à agência de fomento CAPES-PROEX (Coordenação de Aperfeiçoamento Pessoal de Nível Superior) pela bolsa. Agradecem também à empresa APERAM South America pelo fornecimento de amostras do aço inox duplex.

\section{REFERÊNCIAS}

1 OLASEINDE, O. A.; MERWE, J. V.; CORNISH, L. Characterization and corrosion behavior of selected duplex stainless steel. Advances in Chemical Engineering and Science, v. 4, p. 89 - 93, 2014.

2 OUTOKUMPU, Handbook stainless steel. Outokumpu Oyj, 2013. 92p.

3 HERRERA, C.; PONGE, D.; RAABE, D. Characterization of microstructure, crystallografic texture and segregation of an as - cast duplex stainless steel slab. Steel Research International, v. 79, n. 6, p. 482-488, 2008.

4 GUNN, R. N. Duplex satainless steel: Microstructure, properties and applications. 3ed. Cambridge: Abington Publishing; 2003. 204p.

5 GOSH, S. K.; MONDAL, S. High temperature ageing behavior of duplex stainless steel. Materials Characterization, v. 59, p. 1776 - 1783, 2008.

6 PADILHA, A. F.; RANDLE, V.; MACHADO, I. F. Microstructure and microtexture during solution nitriding to produce austenitic case on ferritic - austenitic duplex stainless steel. Materials Science and Technology, v. 15, p. 1015 - 1018, 1999.

$7 \quad$ KAHARAN, T.; EMRE, H. E.; TÜMER, M.; KAÇAR, R. Strengthening of AISI 2205 duplex stainless steel by strain ageing. Materials \& Design. v. 55, p. 250 - 256, 2014.

8 ARMAS, I. A. Duplex stainless steel: Brief history and some recent alloys. Recent Patents on Mechanical Engineering, v. 1, p. 51 - 57, 2008.

9 CHARLES, J. Duplex stainless steel, a review after DSS' 07 held in GRADO Conference Maastricht. 2007

10 FRECHARD, S.; MARTIN, F.; CLEMENT, C.; COUSTY, J. AFM and EBSD combined studies of plastic deformation in a duplex stainless steel. Materials Science Engineering A, v. 418, p. 312 - 319, 2006.

11 CHAN, K. W.; TJONG, S. C. Effect of secondary phase precipitation on the corrosion behavior of duplex stainless steel. Materials, v. 7, p. 5268 - 5304, 2014.

12 CRISTINI, S. I.; SACCHI, B.; GUERRINE, E.; TRASATTI, S.; TRASATTI, S. P. Detection of sigma phase in $22 \%$ Cr duplex stainless steel by eletrochemical methods. Russian Journal of Electrochemistry, v. 46, n. 10, p. 1094 - 1100, 2010.

13 ESCRIBA, D. M.; MORRIS - MATERNA, E.; PLAUT, R. L.; PADILHA, A. F. Chi - phase precipitation in a duplex stainless steel. Materials Characterization, v. 60, p. 1214 1219, 2009. 
14 NILSON, J.O. Super duplex stainless steel. Materials Science and Technology, v. 8, p. $685-700,1992$.

15 ROSSO, M.; PETER, I.; SUANI, D. About heat treatment and properties of duplex stainless steels. Journal of Achievements in Materials and Manufacturing Engineering, v. 59, n. 1, p. 26 - 36, 2013.

16 ELMER, J. W.; PALMER, T. A.; SPECHT, E. D. Direct observations of sigma phase formation in duplex stainless steel using in - situ synchrotron $x$ - ray diffraction. Metallurgical and Materials Transactions A, v. 38A, p. 464 - 475, 2007.

17 CHEN, T. H.; YANG, J. R. Effects of solution treatment and continuous cooling on $\sigma-$ phase precipitation in a 2205 duplex stainless steel. Materials Science ang Engineering A, v. 311 , p. $28-41,2001$

18 LI, J.; WU, T.; RIQUIER, Y. $\sigma$ Phase precipitation and its effect on the mechanical properties of a duplex stainless steel. Materials Science and Engineering A, v. 174, p. 149 - 156, 1994.

19 WEN, S. Metallurgical evaluation of cast duplex stainless steel and their weldments. 2001. 221p. Master thesis. (Mater of Science) - The University of Tennessee, Knoxville, 2001.

20 ZHANG, W.; JIANG, L. Z.; GAO, J. Q.; SONG, H. M.; HU, J. C. Study of precipitation in 2101 duplex stainless steel. Materials Science and Technology, v. 26, n. 5, p. 515 521, 2010.

21 HSIEH, C. C.; WU, W. Overview of intermetallic sigma $(\sigma)$ phase precipitation in stainless steel. ISRN Metallurgy, v. 2012, p. 1 - 16, 2012.

22 TAVARES, S. S. M.; PARDARL, J. M.; GUERREIRO, J. L.; GOMES, A. M.; SILVA, M. R. Magnetic detection of sigma phase in duplex stainless steel UNS 31803. Journal of Magnetism and Magnetic Materials, v. 322, p. L29 - L33, 2010.

23 VILLANUEVA, D. M. E.; JUNIOR, F. C. P.; PLAUT, R. L.; PADILHA, A. F. Comparative study on sigma phase precipitation of three types of stainless steels: austenitic, superferritic and duplex. Materials Science and Technology, v. 22, n. 9, p. 1098 - 1104, 2006.

24 LLORCA - ISERN, N.; LOPEZ - LUQUE, H.; LOPEZ - JIMENEZ, I.; BIEZMA, M. V. Identification of sigma and chi phase in duplex stainless steel. Materials Characterization, v. 112, p. 20 - 29, 2016.

25 CALLIARI, I.; BREDA, M.; RAMOUS, E.; MAGRINI, M.; STRAFFELINI, G. Effect of isothermal heat treatments on duplex stainless steels impact toughness. In: Convegno Nazionale IGF XXII, 2013, Roma, Itália. Anasis...Roma, Luglio, 2013, p. 56 - 65. 